\title{
BMJ Open Efficacy and economic evaluation of delivery of care with tele-continuous EEG in critically ill patients: a multicentre, randomised controlled trial (Tele-cRCT) study protocol
}

\author{
Chusak Limotai (D) , ${ }^{1}$ Atiporn Ingsathit, ${ }^{2}$ Kunlawat Thadanipon, ${ }^{2}$ \\ Oraluck Pattanaprateep, ${ }^{2}$ Anuchate Pattanateepapon, ${ }^{2}$ \\ Kammant Phanthumchinda, ${ }^{3}$ Nijasri C Suwanwela, ${ }^{1}$ Iyavut Thaipisuttikul, ${ }^{1}$ \\ Kanokwan Boonyapisit, ${ }^{4}$ Ammarin Thakkinstian ${ }^{2}$
}

To cite: Limotai $C$, Ingsathit A, Thadanipon $\mathrm{K}$, et al. Efficacy and economic evaluation of delivery of care with tele-continuous EEG in critically ill patients: a multicentre, randomised controlled trial (Tele-cRCT) study protocol. BMJ Open 2020;10:e033195. doi:10.1136/ bmjopen-2019-033195

- Prepublication history and additional material for this paper are available online. To view these files, please visit the journal online (http://dx.doi. org/10.1136/bmjopen-2019033195).

Received 26 July 2019 Revised 28 December 2019 Accepted 14 February 2020

Check for updates

(C) Author(s) (or their employer(s)) 2020. Re-use permitted under CC BY-NC. No commercial re-use. See rights and permissions. Published by BMJ.

For numbered affiliations see end of article.

Correspondence to Dr Atiporn Ingsathit; atiporn.ing@mahidol.ac.th

\section{ABSTRACT}

Introduction Some critically ill patients are confirmed by continuous electroencephalography (cEEG) monitoring that non-convulsive seizure (NCS) and/or non-convulsive status epilepticus (NCSE) are causes of their depressed level of consciousness. Shortage of epilepsy specialists, especially in developing countries, is a major limiting factor in implementing $\mathrm{CEEG}$ in general practice. Delivery of care with tele-continous EEG (tele-cEEG) may be a potential solution as this allows specialists from a central facility to remotely assist local neurologists from distant areas in interpreting EEG findings and suggest proper treatment. No tele-cEEG programme has been implemented to help improve quality of care. Therefore, this study is conducted to assess the efficacy and cost utility of implementing tele-cEEG in critical care.

Methods and analysis The Tele-cRCT study is a 3-year prospective, randomised, controlled, parallel, multicentre, superiority trial comparing delivery of care through 'TelecEEG' intervention with 'Tele-routine EEG (Tele-rEEG)' in patients with clinical suspicion of NCS/NCSE. A group of EEG specialists and a tele-EEG system were set up to remotely interpret EEG findings in six regional government hospitals across Thailand. The primary outcomes are functional neurological outcome (modified Rankin Scale, $\mathrm{mRS}$ ), mortality rate and incidence of seizures. The secondary outcomes are cost utility, length of stay, emergency visit/readmission, impact on changing medical decisions and health professionals' perceptions about tele-cEEG implementation. Functional outcome (mRS) will be assessed at 3 and 7 days after recruitment, and again at time of hospital discharge, and at 90 days, 6 months, 9 months and 1 year. Costs and health-related quality of life will be assessed using the Thai version of the EuroQol-five dimensions-five levels (EQ-5D-5L) at hospital discharge, and at 90 days, 6 months, 9 months and 1 year.

Ethics and dissemination This study has been approved by the ethics committees of the Faculty of Medicine, Chulalongkorn University, and of Ramathibodi Hospital, Mahidol University, and registered on Thai Clinical Trials Registry. The results will be disseminated in a peerreviewed journal.
Strengths and limitations of this study

- This study is the first to assess the efficacy and cost utility of implementing tele-continuous electroencephalography (tele-cEEG) in critical care.

- This study is also among the very few studies assessing the efficacy of $\mathrm{cEEG}$ on functional outcome and mortality.

- This study is limited as it implements tele-cEEG only in advanced level hospitals in distant areas, and as such the results cannot be generalised and applied to smaller scale hospitals where neurologists are not available and drug items and/or investigations are limited.

- Blinding will not be possible due to the nature of the study intervention (either tele-routine EEG or telecEEG), so bias from outcome ascertainments might be present.

Trial registration number TCTR20181022002; preresults.

\section{INTRODUCTION}

Status epilepticus (SE) is a life-threatening medical and neurological emergency requiring prompt recognition and treatment. A recent meta-analysis including 43 studies reported a pooled crude annual incidence rate of SE of 12.6 per 100000 (95\% CI 10.0 to 15.3$).^{1}$ The pooled case fatality rate and the pooled crude annual mortality rate of SE were $14.9 \%$ (95\% CI 11.7 to 118.7 ) and 0.98 per 100000 (95\% CI 0.74 to 1.22 ), respectively. ${ }^{1}$ Based on the National Database of Thailand during the 2010 fiscal year, the SE rate in Thailand was 5.10 per 100000 population, with a mortality rate of 0.6 per 100000 population. ${ }^{2}$ 
SE can manifest with either overt convulsive movements or subtle/no overt convulsion. The former and the latter are known as 'convulsive status epilepticus (CSE)' and 'nonconvulsive status epilepticus (NCSE)', respectively. In practice, electroencephalography (EEG) recording is required to help in the diagnosis of non-convulsive seizure (NCS)/NCSE; otherwise, it may be underrecognised and left untreated. ${ }^{3}$ Our recent meta-analysis revealed that continuous EEG (cEEG) is significantly better than routine EEG (rEEG) in helping detect NCS/ NCSE. ${ }^{4}$ The overall prevalence of NCS/NCSE is $15.6 \%$ in critically ill patients, but higher in patients with postconvulsive SE $(32.9 \%)$, central nervous system (CNS) infection $(23.9 \%)$ and postcardiac arrest $(22.6 \%) .{ }^{4}$ Evidence of systemic complications and neurological consequences has been clearly demonstrated in $\mathrm{CSE},{ }^{5}$ but remains unclear for NCS/NCSE. Previous observational studies did not provide clear results as to whether the unfavourable outcome of study patients was a direct consequence of NCS/NCSE or a result of other potential confounding factors, that is, patient characteristics, aetiology and treatment. ${ }^{67}$ As a result, the aggressiveness to treat patients with NCS/NCSE is unknown and varies among treating physicians. ${ }^{6}$

Although EEG recording is necessary in detecting NCS/NCSE, its routine use, particularly cEEG monitoring, remains an issue because it is costly and requires specialists to interpret the findings. ${ }^{3}$ Due to shortage of epilepsy specialists, especially in developing countries, cEEG implementation in general practice is limited. Delivery of care with a telehealth system ${ }^{8}$ may be a promising solution to this problem as it allows specialists to remotely assist general physicians from distant areas in interpreting EEG findings and suggest proper management. Until now, no study has shown how telecontinuous EEG (tele-cEEG) improves quality of care particularly for patients with SE. By doing this, we can at the same time prospectively assess the benefits of tele-cEEG and the neurological consequences of NCS/ NCSE.

The Tele-cRCT study is a multicentre, randomised controlled trial (RCT). With an RCT design, the efficacy of tele-cEEG implementation will be determined with valid results, since potential confounding factors will be balanced and adjusted between two groups of comparison. Alongside economic evaluation of tele-cEEG, cost utility analysis (CUA) will also be performed to determine if it can be introduced to the community and adopted in routine practice.

\section{METHODS AND ANALYSIS}

This study protocol followed the Standard Protocol Items: Recommendations for Interventional Trials (SPIRIT) (see SPIRIT checklist in online supplemental document $1)$.

\section{Study design and setting}

The Tele-cRCT is a 3-year prospective, randomised, controlled, parallel, multicentre, superiority trial comparing delivery of care through 'Tele-cEEG' intervention with tele-routine EEG ('Tele-rEEG') in patients with clinical suspicion of NCS/NCSE. We have currently conducted a pilot study in some study hospitals to test the feasibility of remote EEG monitoring and the whole process of data collection. A group of EEG specialists and a tele-EEG system were set up to remotely interpret EEG in the study hospitals, consisting of six regional government hospitals across Thailand. All six study hospitals have met our eligibility criteria: (1) regional hospitals defined according to the Ministry of Public Health of Thailand as hospitals in service plan A (advanced level hospital) with the capability to treat patients who require advanced and sophisticated technology; (2) hospitals with surgical or medical intensive care units (ICUs) which are run by qualified medical professionals and with sufficient requisite medical equipment in the ICUs, corresponding to any level of three-tiered system ICUs proposed by the American College of Critical Care Medicine'; (3) hospitals with at least two portable EEG machines available and with the capability to operate EEG recording in the ICUs or wards; and (4) hospitals with neurologists who are capable of treating SE with available necessary medications recommended by the 2016 American Epilepsy Society guideline ${ }^{10}$ and with the capability of doing aetiology work-up of SE, as suggested by the 2012 Neurocritical Care Society, ${ }^{3}$ but (5) no qualified epileptologists to interpret the EEG and (6) with cEEG monitoring not part of the hospital's routine service.

A specialist team will assist both intervention (telecEEG) and control (tele-rEEG) arms in interpreting EEG findings and suggest appropriate treatment in order to standardise a 'specialist factor' which might affect study outcomes. It should be noted that the EEG recording, even rEEG, is underutilised in Thailand due to a severe shortage of epileptologists and neurologists who are comfortable and confident in interpreting EEG findings. The study flow is shown in figure 1 .

\section{Study objectives}

Between the intervention (tele-cEEG) and the control (tele-rEEG) arm, our primary objective is to compare efficacy in terms of functional outcomes (modified Rankin Scale, mRS) and mortality rate assessed at 3 and 7 days after recruitment, at discharge, and at 90 days, 6 months, 9 months and 1 year after hospital discharge, as well as detection rate of seizures during hospitalisation. The secondary objective is to compare efficacy in terms of ICU/hospital length of stay, emergency/readmission, cost utility and impact on changing medical decisions, as well as healthcare professionals' perceptions of tele-EEG implementation.

\section{Screening and randomisation}

A dedicated nurse in each study hospital screens for eligible patients in every new admission or new neurology 


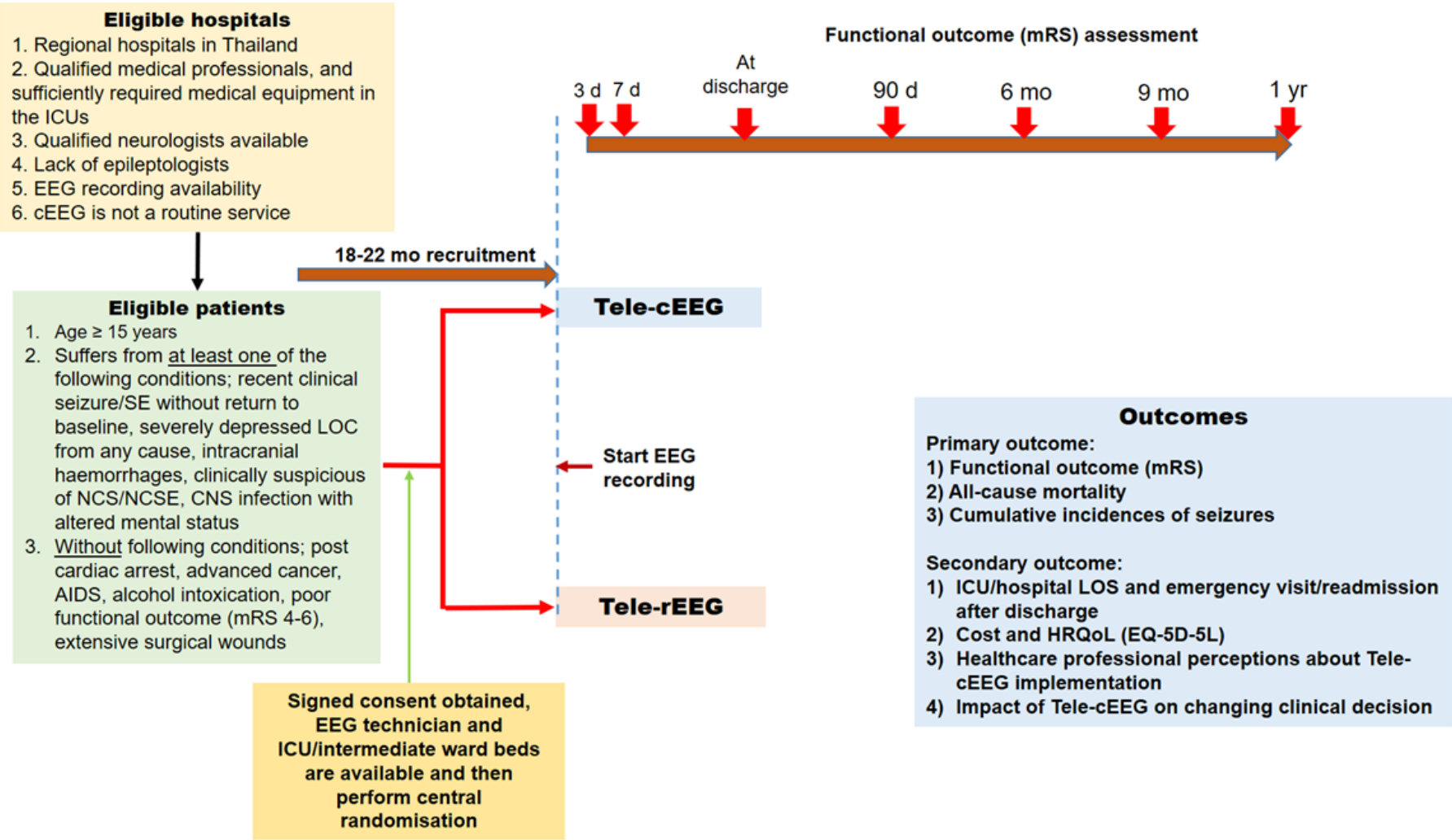

Figure 1 Study flow. cEEG, continuous EEG; CNS, central nervous system; EEG, electroencephalography; EQ-5D-5L, EuroQol-five dimensions-five levels; HRQoL, health-related quality of life; ICU, intensive care unit; LOC, loss of consciousness; LOS, length of stay; mRS, modified Rankin Scale; NCS, non-convulsive seizure; NCSE, non-convulsive status epilepticus; rEEG, routine EEG; SE, status epilepticus.

consultation from adult ICUs or medical or surgical wards to see whether or not potential study subjects fulfil one of the five conditions indicated in the inclusion criteria listed in box 1. Eligibility is then confirmed with a neurologist at the study site. If eligibility criteria were fulfilled, a nurse will provide study information to patients or relatives and then request for signed informed consent. The nurse will then log in and fill out the study web-based screening form. If the patient is eligible, the system will automatically run central randomisation and assign the patient to a study intervention (tele-cEEG vs tele-rEEG) along with the patient's subject identification number for the study. Block randomisation will be applied. Since this is not a double-blind study, healthcare teams will not be blinded to the intervention, and to protect the integrity of the randomisation process random selection of block size will be performed prior to randomly selecting the patient. The block sizes will be $4,6,8$ and 10 . The ratio of the intervention to control is 1:1. Statisticians at the central site will generate random sequences of assigned intervention using STATA V.15.0. The study flow and the investigator's role are shown in online supplementary figure 1.

\section{Allocation concealment}

To prevent selection bias, central randomisation will be applied to conceal allocation sequence from those assigning participants to intervention groups until the time of assignment.

\section{Blinding}

As the nature of assigned intervention is different and easy to recognise (ie, cEEG (prolonged) vs rEEG (short) recording), participants will not be blinded to the intervention assigned. Healthcare teams including physicians and nurses will also not be blinded because they will be involved in patient care using either cEEG or rEEG. However, dedicated outcome assessors will be blinded to patient allocations.

\section{Intervention}

This study consists of two arms which apply two different interventions: one with tele-cEEG (24-hour monitoring, intervention arm) and the other with tele-rEEG (30 min monitoring, control arm) (see figure 2). Since the important study outcomes are functional outcomes and mortality after SE, a specialist team will assist the control arm (tele-rEEG) in interpreting EEG findings and suggest appropriate treatment in order to standardise a specialist factor which might affect the outcomes.

\section{Tele-EEG system and database}

The central facility for the tele-EEG system/EEG database and the patient's database were, respectively, set up at the Chulalongkorn Comprehensive Epilepsy Center 
Box 1 Inclusion and exclusion criteria for patient enrolment

\section{Inclusion criteria.}

- Adult patients, aged $\geq 15$ years, who are admitted to surgical or medical ICUs or wards.

- Suffering from at least one of the five conditions as recommended by the 2012 Neurocritical Care Society*, as well as corresponding with the results of our meta-analysist to be highly associated with NCS/NCSE.

- Recent clinical seizure/status epilepticus without return to baseline (prestatus):

- If receiving sedative medication: at $>10$ min after clinical seizure/ SE ends, the patient's GCS score does not return to baseline.

- If not receiving sedative medication: at 2 hours after clinical seizure/SE ends, the patient's GCS score does not return to baseline.

- Severely depressed consciousness from any cause (except for TBI, SAH and ICH) with GCS score $\leq 8$.

- Intracranial haemorrhages with any of the following:

- TBI with GCS score 6-12.

- SAH with Hunt and Hess classification grade $\leq \mathrm{IV}$ or GCS score $>5$. - ICH with score $\leq 3$.

- Suspected NCS/NCSE in patients with altered mental status (indeterminate cause).

- CNS infection with altered mental status.

- Patients and/or their relatives willing to participate in the study and have given signed informed consent.

- Patients or caregivers, defined as the main person other than health, social or voluntary care provider who can provide functional outcome data after discharge.

Exclusion criteria.

- Patients with postcardiac arrest.

- Patients with advanced stage cancer (stage IV).

- Patients with AIDS (CD4 count $<200$ cells $\times 10^{6} / \mathrm{L}$ or with certain opportunistic infections).

- Patients with alcoholic intoxication with/without delirium tremensł.

- Patients with poor functional outcome at preadmission state (mRS score 4-6).

- Patients with extensive lacerations, skin lesions or surgical wound where the electrode placement cannot be applied.

\section{*Brophy et al. ${ }^{3}$}

†Limotai et al. ${ }^{4}$

¥These patients are excluded due to the fact that there are a large number of these types of patients in rural areas of Thailand who may significantly outweigh other types of patients included, where there has been no reported magnitude of its association with NCS/NCSE.

CNS, central nervous system; GCS, Glasgow Coma Scale; ICH, intracerebral haemorrhage; ICU, intensive care unit; mRS, modified Rankin Scale; NCS, nonconvulsive seizure; NCSE, non-convulsive status epilepticus; SAH, subarachnoid haemorrhage; SE, status epilepticus; TBI, traumatic brain injury.

of Excellence (CCEC) and the Department of Clinical Epidemiology and Biostatistics, Ramathibodi Hospital (Rama CEB). Two separate EEG review systems will be set up: one for real-time review using the TeamViewer software and the other for offline review using EEG data uploaded on cloud storage. For offline review, EEG data uploaded on cloud storage will be downloaded into the EEG database server at CCEC on a daily basis. Each EEG specialist in charge can connect to the EEG machine

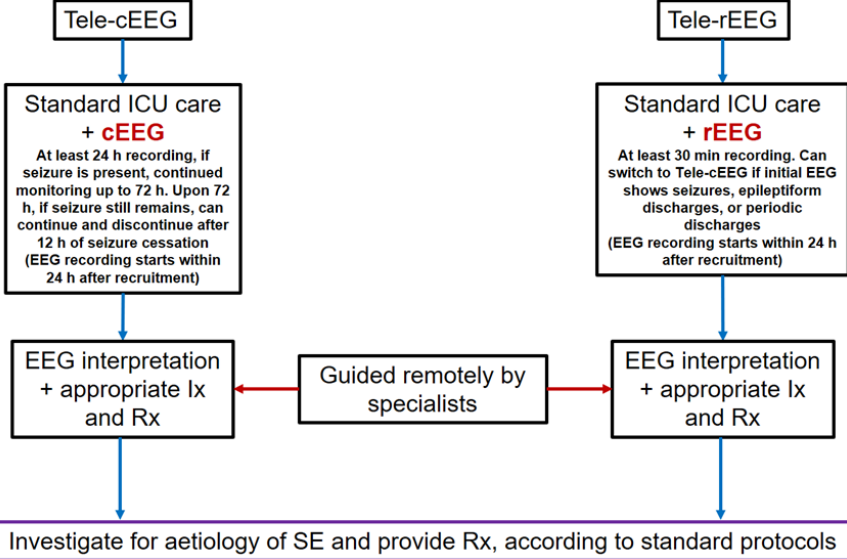

Figure 2 Implementation of study interventions. cEEG, continuousEEG; EEG, electroencephalography; ICU, intensive care unit; Ix, investigation; rEEG, routineEEG; $R x$, treatment; SE, status epilepticus.

at study sites and the EEG server at CCEC for real-time and offline review, respectively, anytime and anywhere via the internet ('De-centralized system') (see figure 3). Password access will be provided for both real-time and offline review.

\section{Methods of conducting tele-EEG}

The EEG recording must be initiated within 24 hours after recruiting (randomisation) patients in both arms (tele-cEEG vs tele-rEEG). Within working hours (from 08:00 to 16:00), an EEG technician will apply the EEG electrodes, and at the same time a specialist in charge on that day will be notified to prepare for the EEG review. After an internet connection has been set up, the integrity of the tele-EEG system will be checked at both ends.

For tele-cEEG, a specialist will periodically report EEG findings using a standard case record form (CRF) every 2, 6 or 12 hours, depending on clinical urgency as

Central site

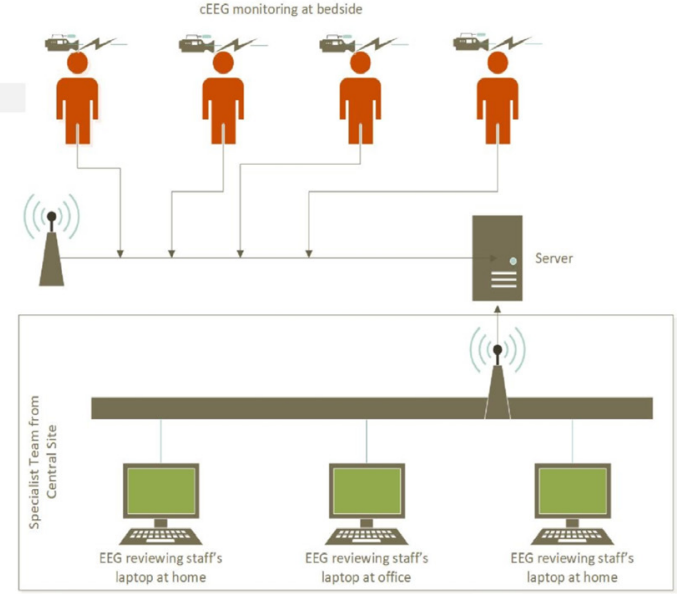

Figure 3 'De-centralized system' of the tele-EEG. Each EEG specialist in charge can connect to the EEG machine at study sites and EEG server at the Chulalongkorn Comprehensive Epilepsy Center of Excellence for real-time and offline review, respectively, anytime and anywhere via the internet. cEEG, continuous EEG; EEG, electroencephalography. 
determined by clinical data and the initial $30 \mathrm{~min} /$ prior EEG findings. EEG will be monitored for at least 24 hours. If seizures are detected, tele-cEEG will be continued and discontinued after 72 hours. However, if seizures are still present at 72 hours, tele-cEEG can be continued and then discontinued after seizure cessation for 12 hours. Continuation of tele-cEEG monitoring after 72 hours will be treated as cointervention (see figure 2).

For tele-rEEG, a specialist will interpret EEG findings and feed back the results using a standard CRF to the treating neurologist at bedside within 2 hours after finishing the EEG study. EEG will be monitored and recorded for $30 \mathrm{~min}$. Switching from tele-rEEG to telecEEG is possible if the initial findings reveal seizures and/or epileptiform activity or periodic discharges. These specific EEG findings were reported by the 2012 Neurocritical Care Society guideline to be highly associated with NCS/NCSE. ${ }^{3}$ In this case, tele-cEEG will be treated as cointervention (see figure 2). Performing additional rEEG in case a clinical concern for ongoing seizure remains is allowed, although once again this will be recorded and treated as cointervention.

In both arms, standard consensus protocols for investigations and management of SE will be followed for all patients. A specialist in charge will discuss the EEG findings with the treating neurologist at bedside and then appropriate management will be provided according to consensus protocols. Flexible connectivity will be used, where specialists who review the EEG can access patient medical information on cloud storage via the internet ('Open communication architecture') (see online supplementary figure 2). Communication between specialists and treating neurologists is limited to traditional telephonic modalities and is functionally outside the tele-EEG system (see online supplementary figure 2).

\section{EEG reviewing organisation}

Nine EEG specialists included in this study are all certified epileptologists with training in either Thailand and/ or North America (USA and Canada). All EEG specialists will be on-call for EEG review. Each on-call duration lasts for 24hours (from 07:00 to 07:00 on the following day). EEG specialists are responsible for reviewing both the cEEG and the rEEG on that day. An EEG specialist will give his/her report to the other EEG specialist on the following day by verbal communication using a unified EEG finding and list of management report forms to ensure continuity of appropriate management.

Standard consensus protocols for investigations and management of SE were developed using a modified Delphi method. ${ }^{11}{ }^{12}$ All nine EEG specialists were invited to perform online Google survey and then face-to-face discussion in order to standardise and make consensus protocols on how to report EEG findings and manage SE. The terminology and definition of the EEG waveforms used in this study will be mainly based on the American Clinical Neurophysiology Society's proposed standardised terminology (2012 version). ${ }^{13}$ A unified EEG report form

\section{Box 2 Primary and secondary outcomes}

\section{Primary outcome.}

- Functional outcomes including poor (mRS score 4-6) versus favourable (mRS score 0-3) functional outcomes and functional decline (ie, $\mathrm{mRS}$ increases at least one score) of the actual scores, in which $\mathrm{mRS}$ will be assessed at 3 and 7 days after starting EEG recording (recruitment), at discharge, and at 90 days, 6 months, 9 months and 1 year.

- ICU/in-hospital case fatality rate during hospitalisation and crude annual mortality rate assessed at 1 year after hospital discharge.

- Cumulative incidence of each type of seizures, that is, pure NCS/ NCSE, combined NCS/NCSE and CS/CSE, and pure CS/CSE, in the intervention and control arms.

Secondary outcome.

- ICU and hospital length of stay.

- Emergency visit and readmission after hospital discharge assessed at 90 days, 6 months, 9 months and 1 year.

- Health-related quality of life, assessed by EuroQol-five dimensionsfive levels (EQ-5D-5L) Thai version, at hospital discharge, and at 90 days, 6 months, 9 months and 1 year.

- Costs assessed at hospital discharge, and at 90 days, 6 months, 9 months and 1 year.

- To assess the impact of change in medical decisions of the treating neurologists at study sites, a structured questionnaire will be assessed immediately after patient recruitment, but prior to knowing the EEG results, and then compared with the actual activities (investigations/treatment) after integrating the EEG findings with other clinical data.

- To assess health professionals' perceptions about tele-cEEG implementation, a structured questionnaire will be evaluated by nurses and neurologists at study sites, assessed at 1 year after conducting the study (see online supplementary table 2).

CS, convulsive seizure; CSE, convulsive status epilepticus; EEG, electroencephalography; ICU, intensive care unit; mRS, modified Rankin Scale; NCS, non-convulsive seizure; NCSE, non-convulsive status epilepticus; telecEEG, tele-continuous EEG.

will be created as part of the web-based CRF. Twenty-three and five EEG tracings with a variety of common EEG findings in critically ill and seizure/SE EEG patterns were prepared and then used to test inter-rater agreement ${ }^{14}$ among seven EEG specialists (see online supplementary table 1). Per cent level of agreements of these parts were, respectively, 79.3 and 79.1, with Gwet's kappa coefficients (95\% CI) of 0.7354 (0.5825 to 0.8883$)$ and 0.7373 (0.3409 to 1.0000$)$, indicating substantial agreements for both parts.

\section{Study outcomes}

The primary and secondary outcomes are listed in box 2 .

\section{Sample size calculation}

The primary outcome used for estimation of sample size is functional outcome measured by mRS. It is dichotomised into favourable (mRS score $0-3$ ) and poor (mRS score 4-6) outcomes. The formula for the estimated number of participants is as follows ${ }^{15}$ :

$$
N=\left(Z_{\propto / 2}+Z_{\beta}\right)^{2} \frac{\pi_{0}\left(1-\pi_{0}\right)+\pi_{1}\left(1-\pi_{1}\right)}{\left(\pi_{0}-\pi_{1}\right)^{2}}
$$


where $\mathrm{n}=$ total number of participants; $\mathrm{Z}_{\alpha / 2}=1.96$; $\mathrm{Z}_{\beta}=0.84 ; \Phi_{0}=$ the true proportions in the control population; and $\Phi_{1}=$ the true proportions in the intervention arm.

As in the previous study by Khawaja et al, ${ }^{16}$ which up to now is the only one available study assessing functional outcomes in critically ill patients who received cEEG monitoring (intervention) and also in those who did not receive cEEG (control), ${ }^{16}$ the proportion of patients with poor outcome (mRS score 3-6) was 0.829 for the control group. If we plan to determine a difference of poor functional outcome of 0.1 (which should be clinically meaningful), and setting an intervention to control ratio of 1:1, with type I and II errors of 0.05 and 0.2 , the estimated sample size will be as follows:

$$
\begin{gathered}
N=(1.96+0.84)^{2} \frac{0.829(1-0.829)+0.729(1-0.729)}{(0.829-0.729)^{2}} \\
=7.84 \frac{(0.142+0.198)}{0.01} \\
=267
\end{gathered}
$$

Assuming a $20 \%$ loss to follow-up, the total number of participants required in each arm is $270+54=324$. In summary, to achieve an $80 \%$ power to detect a $10 \%$ reduction of poor outcomes at a $5 \%$ level of significance (twosided), we require 324 participants in each arm, resulting in 648 participants in total.

\section{Patient recruitment}

A pilot study will be performed to assess whether there will be any recruitment issues in the designated study hospitals. The initial recruitment plan is $10-15$ patients per month from each hospital. After the formal pilot study, this plan may be changed according to actual recruitment rate of each hospital. However, the principal investigator (PI) and/or the coordinator nurse at the central site (CCEC) recruitment centres will be continuously monitoring and encouraging patients to join the study via telephone reminder. To prevent bias related to predominantly recruiting participants from one particular study site, actual recruitment rates from the pilot study will be used to weigh the limit of recruitment from each hospital.

\section{Patient and public involvement}

Neither patients nor the public have been involved in the design of the Tele-cRCT study. The results of the TelecRCT study will be available at https://clinicaltrials.in. th/ both to patients and the general public. Assessment of the burden of the intervention has not been foreseen in the present study.

\section{Data collection and data statement}

CRFs were created according to data on study variables, intervention and outcomes. These were divided into nine parts and created in paper-based forms, except for patient screening and EEG finding which were both created in web-based CRFs (see online supplementary table 3). Timing of data collection is shown in online supplementary table 4 . After obtaining ethics committee approval from each study hospital and signed consent from patients or caregivers, the PI will access patient information and collect patient data from respective study hospitals.

Participant neurologists assigned as sub-PIs in each study hospital will help facilitate access of archived raw data. Study variables and outcomes will be collected during the enrolment period after randomisation and CRFs will be filled in. Independent outcome assessors (either sub-PIs or coordinator nurses at study hospitals) will assess the primary and secondary outcomes.

\section{Data management}

Conversion of the paper and web-based CRFs into an electronic database (EpiData V.3.1, The EpiData Association, Odense, Denmark) is planned. Data entry will be assigned to two data entry staff. Patient database files will be kept in a personal computer at Rama CEB and also backed up in the PI's notebook. These two computers require passwords to access the database. Scheduled site visits for data audits will be arranged for each participant hospital every 1-2 months during the first 6 months and then every 3 months. To ensure appropriate intervention delivery, all completed competency assessment tools will be returned to the PI and will be included as a standard monitoring report to the Data and Safety Monitoring Board (DSMB). Manual, interactive and batch checking methods will be used to ensure completeness and correctness of data. To maintain high-quality data, regular meetings between data collectors and data entry staff will be arranged on a monthly basis to check for data correctness and give feedback.

\section{Data analysis plan}

\section{Descriptive statistics}

Baseline characteristics between tele-cEEG and tele-rEEG arms are presented as mean with SD or median with IQR for continuous data depending on the distribution of data. For categorical data, frequency and percentage are presented. To compare the characteristics of patients between the groups, Pearson's $\chi^{2}$ or Fisher's exact test will be applied for categorical data. Student's t-test or MannWhitney test for normal and non-normal distributed continuous data will be used.

\section{Imputation}

Imputations will be performed using STATA V.15.0 software. Missing data will be explored to assess whether distribution of missing data is missing at random; if not, this is said to be non-ignorable. Multiple imputation (MI) will be applied. The number of imputations will be determined by percentage of missing values and MI performance, ${ }^{17}$ reflected by relative variance increase and fraction of missing information values.

\section{Analytical statistics}

Statistical methods will depend on how the outcomes are being measured and the type of outcomes, either 
dichotomous or continuous, as summarised in online supplementary table 5 . With regard to time to event data analysis of functional outcome (mRS), the start date will be set as the date of starting the EEG recording. Patients will be initially stratified to having poor (mRS score 4-6) versus favourable (mRS score $0-3$ ) outcome at discharge. These two groups will be analysed separately. In the group with an initial poor outcome, time to first ever favourable outcome will be analysed, whereas in the group with initial favourable outcome time to first ever poor outcome will be estimated. Since death will be treated as competing risk, probabilities of developing interested events (poor or improved outcome) will not be independent from probability of death, in which case a cumulative incidence function ${ }^{18}$ will be used instead of Kaplan-Meier method. The end date will be the date at the end of study (1 year after hospital discharge), date of developing interested events, date of having competing risks and date of loss to follow-up. Either cause-specific or subdistribution proportional hazard model will be used to estimate effect sizes, and depends on whether or not the intervention (telecEEG) has an effect on the hazards of competing risks (death). ${ }^{19}$ If it has no effect, a cause-specific proportional hazard model with csHR will be reported. However, in the event of an effect, a subdistribution model with sub-HR will be reported.

Multilevel analysis with mixed-effects models using maximum likelihood estimation will be applied to assess intervention effects ${ }^{20}$ on functional outcome. A mixedeffect model will be constructed as follows: First, intervention variable will be fitted as fixed effect and random effect in a multilevel equation with poor/favourable function as the outcome variable. Second, a random effect of intervention will be constructed. A likelihood ratio will be applied to compare whether considering intervention effect as random will improve model fitting. Adjusted OR along with its $95 \%$ CI will be estimated.

Even if randomisation is used, all of the prognostic factors may not be perfectly balanced. Covariate adjustment will be used in the analysis of the primary and secondary outcomes to minimise the effect of covariate imbalance. The following important covariates at baseline which may influence the study outcomes (ie, functional outcome and mortality) will be adjusted: age ( $\geq 60$ vs $<60$ years), ${ }^{21} 22$ aetiology of SE (acute vs chronic aetiology), ${ }^{22}$ severity of the disease within 24 hours of admission (higher vs lower Acute Physiology and Chronic Health Evaluation IV (APACHE IV)/Simplified Acute Physiology Score II (SAPS II) / Glasgow Coma Scale (GCS) scores) ${ }^{23}$ and history of epilepsy/antiepileptic drug use. The specific adjustment procedure depends on the type of covariate being adjusted for and the type of outcome being analysed. In this study, both primary response variables (primary outcomes) and important covariates are categorical (ie, age, aetiology of SE, severity of disease), so 'a stratified analysis' taking the form of a Mantel-Haenszel statistics will be used. Study participants will be subdivided into smaller, more homogeneous groups, or strata will be used. A comparison of study groups will be done within each stratum and then averaged over all strata to achieve a summary result for the outcome.

\section{Prespecified subgroup analysis}

We plan to perform a subgroup analysis on covariates which potentially affect modifiers of the intervention effects. This may help identify the specific population most likely to benefit from or to be harmed by tele-cEEG. The following subgroup analysis will be assessed: older age ( $\geq 60$ years) versus younger ( $<60$ years), and patients with severe diseases (ie, higher score) versus milder severity (ie, lower score). This will be based on APACHE IV, SAPS II and GCS within 24 hours of enrolment; indications for EEG study (prior clinical seizure/SE without recovery, coma, severely depressed loss of consciousness, intracranial haemorrhages, suspicious NCS/NSCE, CNS infection, and presence of epileptiform discharges or periodic pattern on initial EEG); higher SE severity score versus lower scores (based on the Status Epilepticus Severity Score and the Epidemiology-based Mortality Score in Status Epilepticus score); and type of SE (ie, pure CSE vs pure NCSE vs combined CSE and NCSE).

\section{Dealing with protocol violation}

We will analyse data using the following methods: (1) intention-to-treat analysis: all participants and their outcomes will be included for primary analysis; (2) as-treated analysis: this will be used in the following cases: (a) patients who are initially randomised to receive telerEEG but are subsequently switched to receive tele-cEEG as initial rEEG revealed seizure/epileptiform and/or periodic discharges, and (b) patients with incorrect intervention allocation administration, for example, patients allocated to tele-cEEG are incorrectly administered telerEEG or vice versa; (3) per-protocol analysis: this analysis refers to inclusion in the analysis of only those patients who strictly adhered to the protocol. Flow of analysis is shown in online supplementary figure 3 .

\section{Economic analysis}

Economic analysis will be done alongside the RCT (trialbased economic evaluation). Costs and outcomes will be collected from all patients. We will perform CUA, which will enable the findings from our study to be compared with other healthcare interventions. We will conduct economic analysis in view of societal perspectives, including billing costs, to assess whether tele-cEEG is economically feasible and worthwhile to implement in the context of Thailand.

\section{Outline of interventions}

Using TreeAge Pro 2016, a decision tree will be created using RCT-based data. This decision tree diagram will help depict choices of intervention, the logical structure of probabilities of conditions which could occur after applying the interventions, and values related to cost and utility associated with consequences related to each condition. Interested events discovered by the study 
interventions (tele-cEEG and tele-rEEG) are pure NCS/ NCSE, combined CS/CSE and NCS/NCSE, pure CS/ CSE, and no seizure. The decision tree diagram is shown in online supplementary figure 4 . Parameters and data sources for probabilities of interested events, cost and utility are shown in online supplementary table 6 .

\section{Cost analysis}

Unit costs of services will be referenced on a price provided by the Center of Essential Information for All Health Officers (2018). All costs will be converted to 2018 values using the Thai consumer price index (Bureau of Trade and Economic Indices, 2018). Lifetime time horizon is a cycle length of 1 year. All costs and outcomes occurring after 1 year will be discounted at a rate of $3 \%$, as recommended in the Thai Health Technology Assessment guideline. ${ }^{24}$

\section{Determining cost-effectiveness}

For primary economic analyses, CUA cost per qualityadjusted life-year (QALY) gained based on EuroQol-five dimensions-five levels (EQ-5D-5L) score will be examined. The EQ-5D-5L is a generic, preference-based measure for which a previous study in Thailand reported coefficients for converting to utility. ${ }^{25}$

$$
\text { QALYs }=\text { number of years lived } x \text { utility }
$$

Utility can range from 0 as worst health state or death, to 1 as best health state or healthy. To convert the EQ-5D-5L quality of life score to utility, we use coefficients from a study by Pattanaphesaj (http://www.hitap.net/documents/89762). ${ }^{25}$

The incremental cost-effectiveness ratio (ICER) will be calculated by the formula below. ${ }^{26}$ The numerator will be the difference of the mean total cost between the intervention (tele-cEEG) and the control (tele-rEEG). The mean total cost will be calculated by dividing the summation of all costs at discharge, 90 days, 6 months, 9 months and 1 year in each patient with the total number of patients. The denominator will be the difference of QALY based on EQ-5D-5L score at 1 year between the intervention and the control.

$I C E R=\frac{\text { Mean }(\text { Total cost Tele }-c E E G)-\text { Mean }(\text { Total cost Tele }-r E E G)}{\text { Median }(Q A L Y \text { Tele }-c E E G)-\text { Median }(Q A L Y \text { Tele }-r E E G)}$

We will also derive 95\% CI for the ICER. If the numerator (cost data) and denominator (quality of life data) of the ICER follow a joint normal distribution, Fieller's method will be used. ${ }^{27}$ However, if either data are nonnormally distributed, a non-parametric bootstrap method will be used..$^{28}$ The combination of $95 \%$ CIs for cost and effect differences will be shown in a graph to demonstrate a 'confidence box' of the cost-effectiveness plane. ${ }^{28}$

For the secondary economic analysis, ICER to represent additional cost per additional point on the mRS will be calculated as below. This will be separately assessed at 3 days and 7 days after starting EEG recording, at discharge, and at 90 days, 6 months, 9 months and 1 year. In each time point, the numerator of the ICER will be the difference of the mean total cost between the intervention and the control. The denominator will be the difference of the median mRS score between the intervention and the control at that time point. Cost-effectiveness plane and cost-effectiveness acceptability curves will be presented.

$$
I C E R=\frac{\text { Mean }(\text { Total cost Tele }-c E E G)-\text { Mean }(\text { Total cost Tele }-r E E G)}{\text { Median }(\text { mRS score in Tele }-c E E G)-\text { Median }(\text { mRS score in Tele }-r E E G)}
$$

\section{Uncertainty analysis}

To handle cost analysis uncertainty, a probabilistic sensitivity analysis using Monte Carlo simulation with 1000 bootstrapping replications will be used. One-way analysis will be applied using tornado diagram.

\section{Analytical statistics}

To test the hypothesis on the differences in costs between the intervention and the control arm, a linear regression with cost as response variable will be performed. Since this study has a large sample size $(>50)$, even cost data are highly skewed. Both linear regression relying on central limit theorem and non-parametric bootstrap methods have been proven to be accurate in estimating the true SEs. ${ }^{29}$ In this study, we will use linear regression for analysis since it is easier to implement. Complete case analysis will also be used to deal with missing data.

\section{ETHICAL CONSIDERATIONS}

The ethical conduct of this study will be monitored by the independent DSMB which is part of the Faculty of Medicine, Chulalongkorn University Ethical Review Board. This is an investigator-generated study performed in full independence of study sponsor from any other funding agencies. This study will comply with the commonly agreed international standards for good practice in research, the Belmont Report. Any important protocol modifications will be reported to the ethics committee of both institutions and the trial registries. English-language examples of the patient consent form are shown in online supplemental document 2.

\section{Author affiliations}

${ }^{1}$ Division of Neurology, Department of Medicine, Chulalongkorn University, Bangkok, Thailand

${ }^{2}$ Department of Clinical Epidemiology and Biostatistics, Mahidol University Faculty of Medicine Ramathibodi Hospital, Bangkok, Thailand

${ }^{3}$ Division of Neurology, Department of Medicine, Mahidol University Faculty of Medicine Ramathibodi Hospital, Bangkok, Thailand

${ }^{4}$ Division of Neurology, Department of Medicine, Mahidol University Faculty of Medicine Siriraj Hospital, Bangkok, Thailand

Contributors CL had full access to all of the data in the study, takes responsibility for the integrity of the data and the accuracy of the data analysis, and contributed to study concept or design, acquisition/analysis/interpretation of data, drafting the manuscript, critical revision of the manuscript for important intellectual content, statistical analysis, and administrative/technical/material support. Al contributed to study concept or design, critical revision of the manuscript for important intellectual content, and study supervision. KT contributed to critical revision of the manuscript for important intellectual content and study supervision. OP contributed to study concept or design, drafting the manuscript, critical revision of the manuscript for important intellectual content and study supervision. AP contributed to study concept or design, critical revision of the manuscript for important intellectual content, administrative/technical/material support and study supervision. KP contributed to critical revision of the manuscript for important intellectual content 
and study supervision. NCS contributed to critical revision of the manuscript for important intellectual content and study supervision. IT contributed to critical revision of the manuscript for important intellectual content and administrative/ technical/material support. KB contributed to critical revision of the manuscript for important intellectual content and study supervision. AT contributed to study concept or design, acquisition/analysis/interpretation of data, drafting the manuscript, critical revision of the manuscript for important intellectual content, statistical analysis and study supervision.

Funding This study is funded by the Thailand Research Fund (grant number RSA6280071).

Disclaimer The funder had no involvement in the study design; in the collection, analysis and interpretation of the data; in writing the report; and in the decision to submit the paper for publication.

Competing interests None declared.

Patient consent for publication Not required.

Ethics approval The Tele-cRCT study protocol has been approved by the ethics committees of the Faculty of Medicine, Chulalongkorn University, and the Faculty of Medicine, Ramathibodi Hospital, Mahidol University.

Provenance and peer review Not commissioned; externally peer reviewed.

Open access This is an open access article distributed in accordance with the Creative Commons Attribution Non Commercial (CC BY-NC 4.0) license, which permits others to distribute, remix, adapt, build upon this work non-commercially, and license their derivative works on different terms, provided the original work is properly cited, appropriate credit is given, any changes made indicated, and the use is non-commercial. See: http://creativecommons.org/licenses/by-nc/4.0/.

ORCID iD

Chusak Limotai http://orcid.org/0000-0002-3136-9199

\section{REFERENCES}

1 Lv R-J, Wang Q, Cui T, et al. Status epilepticus-related etiology, incidence and mortality: a meta-analysis. Epilepsy Res 2017;136:12-17.

2 Tiamkao S, Pranbul S, Sawanyawisuth K, et al. A national database of incidence and treatment outcomes of status epilepticus in Thailand. Int J Neurosci 2014;124:416-20.

3 Brophy GM, Bell R, Claassen J, et al. Guidelines for the evaluation and management of status epilepticus. Neurocrit Care 2012;17:3-23.

4 Limotai $\mathrm{C}$, Ingsathit $\mathrm{A}$, Thadanipon $\mathrm{K}$, et al. How and whom to monitor for seizures in an ICU: a systematic review and metaanalysis. Crit Care Med 2019;47:e366-73.

5 Sutter R, Dittrich T, Semmlack S, et al. Acute systemic complications of convulsive status Epilepticus-A systematic review. Crit Care Med 2018;46:138-45.

6 Cockerell OC, Walker MC, Sander JW, et al. Complex partial status epilepticus: a recurrent problem. J Neurol Neurosurg Psychiatry 1994;57:835-7.

7 Adachi N, Kanemoto K, Muramatsu R, et al. Intellectual prognosis of status epilepticus in adult epilepsy patients: analysis with Wechsler adult intelligence Scale-revised. Epilepsia 2005;46:1502-9.

8 Young LB, Chan PS, Lu X, et al. Impact of telemedicine intensive care unit coverage on patient outcomes: a systematic review and meta-analysis. Arch Intern Med 2011;171:498-506.
9 Haupt MT, Bekes CE, Brilli RJ, et al. Guidelines on critical care services and personnel: recommendations based on a system of categorization of three levels of care. Crit Care Med 2003;31:2677-83.

10 Glauser T, Shinnar S, Gloss D, et al. Evidence-Based guideline: treatment of convulsive status epilepticus in children and adults: report of the Guideline Committee of the American Epilepsy Society. Epilepsy Curr 2016;16:48-61.

11 Kelly SE, Moher D, Clifford TJ. Defining rapid reviews: a modified Delphi consensus approach. Int J Technol Assess Health Care 2016;32:265-75.

12 Slade SC, Dionne CE, Underwood M, et al. Standardised method for reporting exercise programmes: protocol for a modified Delphi study. BMJ Open 2014;4:e006682.

13 Hirsch LJ, LaRoche SM, Gaspard N, et al. American clinical neurophysiology Society's standardized critical care EEG terminology: 2012 version. J Clin Neurophysiol 2013;30:1-27.

14 Klein D. Implementing a general framework for assessing interrater agreement in Stata. Stata J 2018;18:871-901.

15 Donner A, Birkett N, Buck C. Randomization by cluster. sample size requirements and analysis. Am J Epidemiol 1981;114:906-14.

16 Khawaja AM, Wang G, Cutter GR, et al. Continuous electroencephalography (cEEG) monitoring and outcomes of critically ill patients. Med Sci Monit 2017;23:649-58.

17 Rubin DB, Schenker N. Multiple imputation in health-care databases: an overview and some applications. Stat Med 1991;10:585-98.

18 Logan BR, Zhang M-J, Klein JP. Regression models for hazard rates versus cumulative incidence probabilities in hematopoietic cell transplantation data. Biol Blood Marrow Transplant 2006;12:107-12.

19 Tai B-C, Wee J, Machin D. Analysis and design of randomised clinical trials involving competing risks endpoints. Trials 2011;12:127.

20 Hubbard AE, Ahern J, Fleischer NL, et al. To Gee or not to Gee: comparing population average and mixed models for estimating the associations between neighborhood risk factors and health. Epidemiology 2010;21:467-74.

21 Rossetti AO, Logroscino G, Milligan TA, et al. Status epilepticus severity score (stess): a tool to orient early treatment strategy. $J$ Neurol 2008;255:1561-6.

22 Leitinger M, Höller Y, Kalss G, et al. Epidemiology-based mortality score in status epilepticus (EMSE). Neurocrit Care 2015;22:273-82.

23 González-Cuevas M, Santamarina E, Toledo M, et al. A new clinica score for the prognosis of status epilepticus in adults. Eur J Neurol 2016;23:1534-40.

24 Chaikledkaew U, Kittrongsiri K. Guidelines for health technology assessment in Thailand (second edition)--the development process. J Med Assoc Thai 2014;97:S4-9.

25 P J. Health-related quality of life measure (EQ-5D-5L): measurement property testing and its preference-based score in Thai population [Doctoral dissertation]. Mahidol University, 2014.

26 Campbell F, Holmes M, Everson-Hock E, et al. A systematic review and economic evaluation of exercise referral schemes in primary care: a short report. Health Technol Assess 2015;19:1-110.

27 Willan AR, O'Brien BJ. Confidence intervals for cost-effectiveness ratios: an application of Fieller's theorem. Health Econ 1996;5:297-305.

28 Drummond MF, Sculpher MJ, Claxton K, et al. Methods for the economic evaluation of health care programmes. Oxford University Press, 2015.

29 Nixon RM, Wonderling D, Grieve RD. Non-Parametric methods for cost-effectiveness analysis: the central limit theorem and the bootstrap compared. Health Econ 2010;19:316-33. 